\title{
Intentando Hacer Grietas en el Sistema Educativo Mexicano: los contrapuntos en los aprendizajes de los estudiantes de la Licenciatura en Planeación del Desarrollo Rural del Centro de Estudios para el Desarrollo Rural (CESDER) en la Sierra norte de Puebla
}

\begin{abstract}
Germán Ortiz Palomeque
'Universidad Intercultural del Estado de Tabasco, Tacotalpa/Tabasco - México

RESUMEN - Intentando Hacer Grietas en el Sistema Educativo Mexicano: los contrapuntos en los aprendizajes de los estudiantes de la Licenciatura en Planeación del Desarrollo Rural del Centro de Estudios para el Desarrollo Rural (CESDER) en la Sierra norte de Puebla. En el presente documento se estudia un modelo de educación superior alternativo y genuino que auspicia de manera sostenible procesos de aprendizaje a través de las experiencias vividas por los estudiantes de la carrera de Planeación del Desarrollo Rural en el Centro de Estudios para el Desarrollo Rural (CESDER), localizado en la Sierra norte de Puebla. Para tal efecto, se empleó el enfoque etnográfico a partir de entrevistas in situ en la que cada uno de los siete estudiantes (tres mujeres y cuatro hombres) describieron cómo han sido sus aprendizajes, las experiencias en su institución educativa y cómo influyeron estos procesos en sus modos de vida. También se realizaron entrevistas en profundidad con dos docentes del CESDER quienes describieron la filosofía de trabajo asumida en esta casa de estudios. Se concluye que la educación bajo el enfoque que le imprime el CESDER sí ha influido en sus modos de vida porque las experiencias de campo reflexionadas de manera crítica en el aula se interrelacionan con las perspectivas docentes y con los saberes de los campesinos para generar comunidades de aprendizaje situado.

Palabras-clave: Enfoque Etnográfico. Comunidades de Aprendizaje. Modos de Vida Rural. Proyectos de Muerte.
\end{abstract}

ABSTRACT - Trying to Make Cracks in the Mexican Educational System: the counterpoints in the learning of the students of the Degree in Rural Development Planning of the Centro de Estudios para el Desarrollo Rural (CESDER) in the North Mountain of Puebla. This document reviews an alternative and genuine higher education model that sustainably supports learning processes through the experiences lived by students of the Rural Development Planning course at the Centro de Estudios para el Desarrollo Rural (CESDER), located in Sierra Norte de Puebla. For this purpose, 
Intentando Hacer Grietas en el Sistema Educativo Mexicano

the ethnographic approach was used based on interviews in situ in which each of the seven students (three women and four men) described how their learning has been, the experiences in their educational institution, and how these processes influenced in their livelihoods. In-depth interviews were also conducted with two CESDER teachers who described the work philosophy assumed in this house of studies. It is found that education under the CESDER approach has influenced their livelihoods because the field experiences critically reflected in the classroom are interrelated with the teaching perspectives, and with the knowledge of the peasants to generate communities of situated learning.

Keywords: Ethnographic Approach. Learning Communities. Rural Livelihoods. Death Projects.

\section{Introducción}

Un viaje a mediados de noviembre del año 2019 hacia el territorio de la sierra norte de Puebla para conocer in situ la misión que realiza el Centro de Estudios para el Desarrollo Rural (CESDER) generó grandes expectativas porque se tenían noticias de sus actividades en apoyo de las comunidades indígenas campesinas de la zona. Así mismo, los textos consultados aumentaron el interés por conocer las experiencias de sus estudiantes y docentes, así que la travesía desde el sureste mexicano hacia la sede del CESDER en el municipio de Zautla se realizaba con el ánimo elevado. De esta manera al arribar al sitio se constató que desde su apertura los promotores de este centro de estudios habían planteado propósitos claros para la ejecución de estrategias y programas de acompañamiento sostenido con el propósito de identificar junto con las comunidades campesinas e indígenas las necesidades educativas y los problemas socioeconómicos con un enfoque territorial. Así, se emprenden acciones de las que derivan planes y programas socioeducativos que potencien las capacidades de los participantes en la línea de impulsar procesos de vida buena rural sustentable en un plano local y regional además de formar sujetos sociales. En ese sentido se han sistematizado diversas experiencias producto del ejercicio de sus funciones de docencia, investigación-acción participativa y de difusión durante casi cuatro décadas (Izquierdo Moreno, 2010; González Forster, 2016; Messina Raymondi; Serrano Arroyo; Comunidad Aguilar, 2017). Por lo tanto, se consideró la pertinencia de documentar las experiencias de aprendizaje entre sus estudiantes para dar a conocer cómo se han sentado las bases para formar al sujeto social crítico como referente de esa formación ciudadana orientada a generar comunidades participativas y resilientes cuyos miembros dispongan de las herramientas fundamentales para actuar en el mediano y largo plazos como agentes de transformación socio territorial en sus comunidades de origen o desde sus centros de trabajo para "hacer grietas en el sistema” (Hernández-Loeza; Manjarrez Muñoz, 2016; Velasco Cruz, 2016; Messina Raymondi; Serrano Arroyo; Comunidad Aguilar, 2017). Lo anterior debe visibilizarse como un indicador de la influencia real del ser y hacer de la entidad educativa en las regiones que atiende por vocación institucional basada en un modelo de educación popular. 


\section{La educación popular en el CESDER}

La educación popular que impulsa el CESDER es un enfoque orientado a crear los cimientos en la formación de sujetos sociales críticos y empoderados para encarar la dinámica capitalista neoliberal que ha generado desigualdades regionales y precarización de los modos de vida campesino en los países económicamente rezagados que enfrentan hoy día el extractivismo de sus recursos naturales por las empresas transnacionales (Veltmeyer; Petras, 2015; CESDER, 2017). En consecuencia, la educación popular y los procesos de aprendizaje que la acompañan, es un enfoque pedagógico dialéctico, ético y crítico que parte de las experiencias de los sujetos sociales para examinarlas colectivamente e incidir en los cambios y acompañamientos necesarios para crear conciencia de su realidad y empoderarlos para transformarla (Messina Raymondi; Serrano Arroyo; Comunidad Aguilar, 2017). El enfoque coadyuva a formar ciudadanía lo cual implica considerar a la educación popular como un proyecto ético-político liberador inspirado en el pensamiento de Paulo Freire, en las reflexiones de John Holloway y de Hugo Zemelman. El hilo conductor de estos pensadores es el sujeto social e histórico en su trayectoria de aprendizaje vivencial: de la experiencia in situ hacia la resignificación y reconceptualización de su modo de vida para un darnos cuenta de lo que me-nos pasa (Berlanga Gallardo, 2011). Por lo tanto, esa resignificación de los modos de vida campesino implica una reflexión profunda acerca de la identidad cultural y el posicionamiento político ideológico de los sujetos sociales desplazando la falsa postura de neutralidad que se difunde profusamente en las escuelas convencionales creadas desde el Estado y por algunas entidades educativas de la sociedad civil. Sin duda que todo proyecto educativo tiene una raigambre político ideológica ya sea orientado hacia la capacitación para administrar el statu quo o formación para incidir sustancialmente en la transformación de las condiciones económicas ambientales y socioterritoriales que enfrentan los núcleos de población rural y urbana marginada (Hernández-Loeza, 2014). En ese sentido es vital estudiar las experiencias de aprendizaje para conocer qué tipo de educación se imparte y cómo incide en los modos de vida de los estudiantes que acuden a los centros de estudios con determinadas expectativas.

\section{Repensando al aprendizaje}

El estudio de las experiencias de aprendizaje en el CESDER obliga a conceptualizar sobre el aprendizaje, su naturaleza, su tipología y, más importante aún, las consecuencias de ese proceso en la sociedad contemporánea a la que algunos teóricos han dado en llamar Sociedad del conocimiento (Ayuste; Gros; Valdivielso, 2012).

Ahora bien, para comprender el estudio del aprendizaje han surgido diversos enfoques acerca de los escenarios, factores, motivaciones, tipos, rol de la memoria humana y las maneras en que aprenden las personas desde la infancia hasta la senectud (Papalia; Wendkos Olds; Dus- 
Intentando Hacer Grietas en el Sistema Educativo Mexicano

kin Feldman, 2019). Así, el aprendizaje es un proceso contextualizado mediante el cual se adquieren actitudes, habilidades y valores que van en el sentido de transformar la conducta de los seres humanos con el propósito de impulsar el desarrollo individual, grupal y, por extensión, propiciar el progreso de una sociedad (García Carrasco et al., 2012). Sobre el tema existen diversas corrientes del pensamiento que colocan sobre la mesa del debate multiplicidad de cuestiones que conllevan una carga ideológica y política (Schunk, 2012; Evans; Reid, 2016). Por ejemplo, Schunk (2012) destaca que el aprendizaje se sustenta en tres criterios básicos:

a) Implica un cambio.

b) Perdura a través del tiempo.

c) Ocurre por medio de la experiencia.

El aprendizaje es inferencial, esto es, en muchos casos no se observa el aprendizaje de forma directa sino mediante sus resultados. Así, cuando se trata de monitorear a las personas para identificar lo que asimilaron durante el proceso de aprendizaje el autor destaca que: "El aprendizaje se evalúa con base en lo que la gente dice, escribe y realiza. Implica un cambio en la capacidad para comportarse de cierta manera, ya que a menudo las personas aprenden habilidades, conocimientos, creencias o conductas sin demostrarlo en el momento en que ocurre el aprendizaje" (Schunk, 2012, p. 4).

El aprendizaje acontece porque se da un proceso de exploración para la comprensión partiendo de una relación entre el sujeto (la persona que tiene facultades intelectivas, actitudes, creencias, identidad étnica y de género, etc.) y el objeto del conocimiento (la realidad material circundante) y en esta interrelación existe una intención del sujeto cognoscente e histórico para develar lo que encuentra a su alrededor -e incluso él mismo como sujeto a explorarse al realizar una introspección- hasta lograr hacer suyas las características de aquello que se ha propuesto entender (Zemelman, 2005). En este proceso de asimilar $l o$ que el objeto/sujeto es, el sujeto cognoscente e histórico encuentra barreras físicas, ideológicas, lingüísticas, semánticas o religiosas que pueden tornar lento y sesgado el proceso de aprendizaje; por lo tanto, esa tarea debe asumirse con un espíritu de auto vigilancia crítica y ética (Dussel, 2016). En ese sentido es crucial un aprendizaje existencial y situado que nos permita entender a otras culturas desde un enfoque más inclusivo en una era de cambios societales provocados por las migraciones del campo a la ciudad y desde las regiones marginadas a los polos de desarrollo industrial (Dietz, 2012). En última instancia, se presentan éxodos masivos entre países en un mundo global por las graves fallas de los actores sociales e institucionales para procurar empleos, educación, alimentación, servicios de salud, vivienda digna y seguridad para sus ciudadanos (Castells; Himanen, 2016). Estos fenómenos plantean nuevos desafíos en la manera de educar a los ciudadanos en una sociedad multicultural que necesita de instituciones enfocadas a considerar en el diseño de sus planes y programas de estudio las complejidades de la realidad contemporánea (Dietz, 2012; Turbino, 2016; Quintar, 2019). 
Ahora bien, dado que el tema de los procesos de aprendizaje nos impele a reflexionar sobre la tarea educativa como proyecto político-de ejercicio de derechos para transitar de una democracia representativa a una democracia sustantiva- en distintos ámbitos, en una difícil travesía desde el núcleo familiar hasta la Universidad, es pertinente reflexionar sobre el papel que juega la cultura política de los sujetos. En ese sentido, la cultura política permite explorar si existen actitudes orientadas al análisis de la situación social y al ejercicio democrático entre los estudiantes de las Universidades. ¿Qué es la cultura política? Es un conjunto de creencias, prácticas y valores que guían las acciones de los miembros de una comunidad en asuntos que afectan su vida en sociedad lo que implica sus vínculos con el Estado y sus instituciones. De acuerdo con un estudioso del tema, la cultura política:

\begin{abstract}
Se forma con valores, actitudes, creencias, sentimientos conocimientos e informaciones, de los ciudadanos respecto de las instituciones, personas (políticos, candidatos, etc.) y procesos políticos, con los cuales calculan su acción política: si apoyan o no a un régimen político, si evalúan favorable o desfavorablemente a un gobierno a alguna institución especifica o los resultados de una política pública determinada, si participan de alguna manera en los procesos políticos o en las organizaciones sociales que realizan la intermediación entre lo social y lo político (Durand Ponte, 2009, p. 121).
\end{abstract}

Esta cultura es el resultado de un prolongado proceso de socialización que inicia desde la infancia en la familia y va complejizándose a medida que las personas interactúan con diversos sujetos sociales, participan en distintos espacios públicos e instituciones. Puede afirmarse que la educación en su vertiente formal contribuye a esa socialización, aunque sigue pendiente la búsqueda de los modelos educativos y los procesos de aprendizaje necesarios para encarar los problemas del presente en los que se observan fenómenos como el cambio climático, conflictos interétnicos, la ocupación militar, el despojo territorial y la limpieza étnica (Pappé, 2015). Estos fenómenos provocan migraciones masivas que entrañan nuevos retos de aprendizaje para todos los sujetos sociales y su relación con las instituciones (Dietz, 2012). Así, la cultura política se va consolidando con múltiples experiencias prácticas de los sujetos sociales en su relación con las instituciones públicas que afectan sus vidas satisfaciendo sus expectativas o generándoles frustración al violentar sus derechos civiles y no responder a sus ingentes demandas (Durand Ponte, 2009).

Ahora bien, en virtud de que la cultura política puede fortalecerse mediante la formación para la resiliencia ciudadana debe advertirse que es una tarea muy compleja porque interpela a los sujetos sociales para que reconozcan la existencia de un problema que es urgente atender (Veltmeyer; Petras, 2015; Evans; Reid, 2016). Esta situación demanda un compromiso de los diversos sujetos sociales lo cual implica una transformación de actitudes, desarrollo de destrezas y fomento de va- 
Intentando Hacer Grietas en el Sistema Educativo Mexicano

lores para la acción crítica, ética, propositiva, reflexiva, responsable y solidaria en contextos multiculturales en donde con frecuencia se observan actitudes discriminatorias, colonialistas, racistas y xenófobas que implican extractivismo de recursos naturales, ocupación y despojo territorial (Pappé, 2015). También se censura a lo diferente, extraño, subalterno, vulnerable y políticamente incorrecto porque se interpela al poder ante sus abusos (Chomsky; Pappé, 2016). Así, debe enfatizarse que tras el velo de la retórica humanista y de seguridad nacional esgrimida por los poderes hegemónicos en la praxis se procede con ambiciones geopolíticas ilegítimas porque violentan el marco jurídico internacional y muestran acciones racistas que apuntan hacia la limpieza étnica y el genocidio (Chomsky; Pappé; Barat, 2015; Pappé, 2018). Por lo tanto, para aprehender esta realidad compleja y multifacética es necesario apoyarse en el concepto de ciudadanía. A pesar de ser un concepto con múltiples significados, la ciudadanía puede definirse "como un conjunto de prácticas (culturales, simbólicas y económicas) y de derechos y obligaciones (civiles, políticos y sociales) que define la membresía de un individuo en una comunidad política" (Sumoano; Nieto, 2015, p. 9). El concepto es apropiado para comprender la compleja dinámica del sujeto social que se posiciona en un contexto globalizador/colonizador en el que existen asimetrías de poder y políticas de exclusión (Pappé, 2018).

Esta breve revisión de la literatura visibiliza la problemática que actualmente se enfrenta en el mundo y constituye una parte importante de los temas abordados por la comunidad de aprendizaje durante los encuentros pedagógicos del CESDER.

\section{La travesía metodológica para entender la misión y la praxis del CESDER}

Las diversas transformaciones del mundo contemporáneo obligan a repensar la misión de las instituciones educativas y los procesos de aprendizaje. En consecuencia, se emprendió esta investigación partiendo de las siguientes preguntas de investigación: ¿Qué es una comunidad de aprendizaje? ¿Cómo se resignifica el modo de vida campesino en las comunidades de aprendizaje del CESDER? ¿Cómo ha sido el proceso de aprendizaje en el CESDER? ¿Cómo evalúan los estudiantes el desempeño de la institución? ¿Qué tendencias educativas se observan? El objetivo general fue describir las experiencias de aprendizaje de los estudiantes del cuarto año de la Licenciatura en Planeación para el Desarrollo Rural. Así, se realizó una revisión bibliográfica sobre los procesos de aprendizaje, cultura política, ciudadanía y la educación superior sustentada en el modelo de educación popular como vía para formar sujetos sociales críticos, emancipados, resilientes y responsables en las tareas de conformar una sociedad incluyente, democrática y tolerante pero no permisiva ante los proyectos hegemónicos excluyentes y depredadores de los recursos naturales (Berlanga Gallardo, 2011; CESDER, 2017). Durante el trabajo de campo se empleó el enfoque etnográfico sugerido por Rockwell (2009) mediante entrevistas a siete estudiantes 
del cuarto año de la Licenciatura en Planeación del Desarrollo Rural elegidos vía muestreo por referencias o "bola de nieve”. Así, se entrevistó al primer sujeto informante y una vez culminado el encuentro éste sugirió a otro colaborador considerando su genuina disposición para participar y su conocimiento del tema que se explora. En un primer acercamiento se realizó el trabajo de campo en el periodo del 21 de noviembre al 16 de diciembre del año 2019. Así mismo, participaron dos docentes como informantes clave. Se empleó un guion de observación y una guía de entrevistas además del diario de campo. En esta etapa fueron fundamentales las reflexiones de Dietz y Álvarez Veinger (2014) que guiaron la realización del trabajo con los sujetos sociales al referirse a la etnografía colaborativa:

En la etnografía colaborativa, el encuentro es entendido como ese escenario en el cual los papeles tradicionales (investigador-investigado) pueden desdibujarse y su clara demarcación pasa a un plano más insignificante al activarse procesos de cointerpretación, donde todas las partes contribuyen activamente a interpretar y construir sentido de lo que sucede en el grupo (Dietz; Álvarez Veinger, 2014, p. 61).

Para realizar el trabajo de campo fue necesario crear un ambiente de camaradería con los estudiantes para generar confianza en el trato. Para el análisis y el procesamiento de las respuestas se elaboraron las siguientes categorías: Aprendizaje significativo, Agrado, Desagrado y Expectativas. Cada encuentro con los estudiantes y docentes fue un conversatorio abierto y franco porque se les garantizó el anonimato asignándoles un nombre ficticio.

\title{
Los contrapuntos entre el discurso y la realidad
}

El CESDER surge como una iniciativa de una organización de la sociedad civil para atender las demandas de grupos de población campesina e indígena tal como lo reseña Hernández-Loeza (2018) en un artículo en el que se documentan las características y modalidades de la educación superior intercultural:

\begin{abstract}
La primera institución en esta modalidad es el Centro de Estudios para el Desarrollo Rural (CESDER), que inició actividades en 1982. En 1989, ingresó la primera generación de la licenciatura en Planeación del Desarrollo Rural, y un año después obtuvo el Registro de Validez Oficial (RVOE). A finales de la década de 1990 un grupo de fundadores del CESDER creó la Universidad Campesina Indígena en Red (UCIRED), desde donde se han impartido diversos posgrados, diplomados, cursos y seminarios (HernándezLoeza, 2018, p. 239).
\end{abstract}

En este proceso de creación de entidades educativas necesarias para impulsar procesos de vida buena se ha transitado por diversas etapas con la participación de los pueblos campesinos e indígenas para 
Intentando Hacer Grietas en el Sistema Educativo Mexicano

manifestar sus necesidades económicas y sociales frente a los embates de las políticas públicas del Estado.

En la etapa del trabajo de campo fueron reveladores los significados que cada colaborador le asignó a las experiencias logradas en cuatro años de labores. Los estudiantes son oriundos de diferentes estados del país. En esta sección se registran algunas narrativas.

Evelia (25 años, alfarera, oriunda de San Miguel Tenextatiloyan) es una de las estudiantes más activas de la Licenciatura en Planeación del Desarrollo Rural, cursa el cuarto año de la especialidad en Economía solidaria. Así, describió sus experiencias de aprendizaje en el CESDER:

La manera de compartir conocimientos pues sí es muy positiva. Sin embargo, en la práctica enfrentamos varias situaciones que no del todo se pueden llevar a cabo, pero tratamos de incidir un poquito más con conocimientos y lograrlo, pero sí cuando es lo práctico hay muchas dificultades. Porque aparte del rol de estudiantes está la de ser parte importante para lograr nuestro sustento para contribuir con la familia. Digamos que la formación sí no las da y sí está encaminada como a la práctica, pero ya en las cosas reales pues sí se nos complica, a veces el ser humano tiene sus contradicciones (Evelia).

Evelia reflexiona sobre el don de compartir con los campesinos lo que se reflexiona en el aula y la práctica contribuye a enriquecer las experiencias para hacernos conscientes del rol que debe desempeñarse en la comunidad de acuerdo al modelo pedagógico del CESDER. Sin embargo, la ruta de ese aprendizaje colectivo no está exenta de obstáculos, como afirma la entrevistada, debido a que se juegan diferentes roles dentro y fuera de los espacios áulicos. El trabajo en las aulas se da cada mes durante seis días de intensas sesiones y se labora en las comunidades de origen tres semanas: se parte de la práctica para retornar a reflexionar en el aula las vivencias, plantear dudas y examinar contradicciones encontradas en el trabajo de campo. Es un proceso de resignificación de lo aprendido en el que se interpela cada fase de aproximación a la realidad en la actividad desarrollada:

Este tipo de educación que recibimos tiene diferentes nombres: educación popular, educación alternativa, educación diferente. Hay que ir redactando, narrando como la parte más detallada, llevar como reporte y teorizar. Vamos a campo y regresamos a reflexionar sobre la teoría y sobre la actividad en campo (Evelia).

Evelia relata que para ella ha sido muy difícil llevar el ritmo de trabajo en su institución educativa pero que le ha ayudado a crecer como persona. De esta manera señala que la formación recibida la ha impulsado a reflexionar sobre su cultura e identidad:

Bueno sí es muy positivo como la parte de la valorización porque desde que me acuerdo existen estos modos de vida como desvalorización de los pobres cuando te dicen esto [desvalorizar tu modo de vida] sientes que no vas a poder sobrevivir. Pero cuando convives con personas de diferentes ámbitos esto va formando experiencia y tus modos de vida. He visto las relaciones de los alfareros en San Miguel, las relaciones de los que hacen 
palma, los que hacen pan, los carpinteros. Es un modo de vida familiar que tiene como su lado favorable y no tan favorable porque estos modos de vida son buenos, pero cuando vas allá afuera -a otro entorno- nos enfrentamos como a un mundo al que debemos estar preparados porque estos modos de vida no en cualquier lado son permisibles (Evelia).

Mientras que para los oriundos de la región pueden ser modos de vida buenos y dignos para los sujetos localizados en otras latitudes tal vez esa manera de vivir la vida sea contraria a sus usos y costumbres generando choques o rechazos a formas muy propias de una cultura más en tiempos de desplazamientos y migraciones.

Por otra parte, los maestros/as contribuyen al análisis y reflexión de los problemas del contexto retomando las experiencias de sus estudiantes y las fortalecen con conceptos teóricos y metodológicos. Se le pregunta a Evelia: ¿Qué aprendizajes significativos estás logrando en el CESDER? En ese sentido la entrevistada estimó la situación que enfrentará a futuro dado que está en la recta final de su carrera profesional:

He tenido como pláticas entre los compañeros y nos preguntamos ¿qué hemos aprendido? Ya estamos terminando la carrera, nos queda como medio año y sentimos que no hemos aprendido, que el mundo se viene y eso. He mencionado con personas con las que tengo la oportunidad de conversar que CESDER más que conocimientos es como la experiencia vivida. ¿2Por qué? Porque un concepto, una palabra, la puedes adquirir en un libro, lo lees, lo analizas y ya lo adquiriste, pero la experiencia que se va formando o las vivencias que vas adquiriendo no sólo es de la profesión sino de la vida misma, la vida propia porque también llegas con la familia, con la comunidad. Y bueno para mí sí ha sido difícil porque si quieres formarte tienes que romper varias barreras, bien la parte de los sentimientos, la parte emocional, se entiende mejor viviéndolo (Evelia).

Las vivencias son potenciadas desde la práctica docente porque en la entidad educativa interesa estudiar al sujeto en su devenir histórico y en el intercambio de saberes desde lo local hasta lo global. En ese tenor es pertinente resignificar las experiencias de aprendizaje de Grecia (24 años, soltera, campesina, Puebla) quien participa activamente en la unidad de producción familiar y en su comunidad realizando actividades de limpia y recolección de café, cultivo de maíz y cría de animales de traspatio. Para ella ha sido muy positivo el proceso de aprendizaje en el CESDER porque se le han dado algunas herramientas metodológicas e instrumentos de investigación para sobrellevar las actividades en su comunidad de origen. Actualmente cursa la especialidad de agroecología y consideró que su estancia en la casa de estudios le ha aportado experiencias significativas para cumplir con éxito en la realización de talleres en zonas urbanas. Así, la primera vez que enfrentó ese desafío fue en la ciudad de Puebla con estudiantes de Universidades privadas. En su relato Grecia ilustra cómo logró superar los escollos encontrados para donar su palabra:

En esa ocasión me sentía tan nerviosa que no sabía cómo iniciar la primera sesión. Eran estudiantes "fifís" de universidades privadas de la capital y pues yo estaba preparada para dar un taller a las comunidades campe- 
Intentando Hacer Grietas en el Sistema Educativo Mexicano

sinas y nunca había trabajado con personas del medio urbano. Así que les pedí me dieran media hora para el recambio de mis diapositivas, mi material didáctico pensado para personas del medio rural y pensar cómo le iba a entrar a la tarea. Por fortuna me surgieron las ideas, las estrategias y los ejemplos que al principio no les hacían sentido a estos estudiantes - eran 12 personas - pero luego me fui dando cuenta que les gustó lo que les estaba compartiendo y al final hasta me invitaron para que de nuevo tuviéramos otro encuentro sobre agroecología y elaboración de composta. Reconozco que en verdad fue algo difícil al principio, pero me ayudó mucho reflexionar sobre los aprendizajes en el CESDER porque las herramientas técnicas y las estrategias para trabajar desde lo lúdico que aquí nos dan me ayudaron a suavizar el encuentro y finalmente tranquilizarme para lograr el aterrizaje de la actividad (Grecia).

La participación de Grecia en las comunidades rurales y urbanas le han dado la oportunidad de conocer otros modos de vida y percibir con sentido crítico las reacciones de los sujetos sociales que interpelan el rol de los modos de vida campesinos desde la perspectiva citadina, pero también le ha permitido valorar el potencial del modo de vida en su comunidad de origen.

Otra de las experiencias notables fue la evaluación del aprendizaje por parte de una pareja de estudiantes (Dámaso y Teresa) quienes incluso narraron algunos claroscuros de la institución. En ese tenor una de las entrevistadas (Teresa, 34 años, casada, promotora de desarrollo comunitario, Puebla) responde al planteamiento que le fue hecho acerca de la naturaleza de los Proyectos de muerte y su estudio en el CESDER:

De hecho, en el primer año hay un módulo especializado sólo en los Proyectos de muerte que son las mineras, las presas hidroeléctricas. Ahí es en donde nos damos cuenta de lo que sucede en la comunidad. Mi comunidad es una zona en donde gran parte de la población, digamos que el $50 \%$, emigra hacia los Estados Unidos. A muchas de las personas las vemos con sus cadenas, anillos, aretes y no sabemos de dónde vienen, qué significado tiene un anillo, un arete para un territorio como Ixtacamaxtitlán. Y desde interiorizar, de escuchar, desde saber cuánta agua se contamina para lixiviar [ lavar el oro], cuántos cerros se deterioran, cuánta flora, cuánta fauna, uno hace conciencia y dice no ya no quiero más esas cosas, ya no las voy a usar. Voy a difundir esta información que es el costo de tener esos objetos, traerlos en nuestro cuerpo y lo que ha significado para algunas personas, para nuestro territorio y para nuestro planeta (Teresa).

Los argumentos de Teresa cobran relevancia si se consideran los costos ambientales por permitir la extracción de minerales en los territorios devastados por las actividades de las empresas mineras en su afán de acumulación y concentración de capital. "Es lo que Harvey llama acumulación por desposesión” (Tetreault, 2015, p. 254).

Por otra parte, se les preguntó icuál ha sido el aprendizaje más significativo en el CESDER? Aunque admitieron que eran planteamientos difíciles de responder porque entrañaba hacer un esfuerzo memorístico “y lo suficientemente sinceros para ser realistas" (Dámaso, 36 años). Al respecto, Teresa destacó lo siguiente: 
Creo que de cada módulo ha habido cosas muy importantes. Sí me resuenan frases, por ejemplo, de que hay que problematizar, de que hay que examinar nuestra vida, examinar nuestros trabajos, en nuestros lugares para poder mejorar nuestra situación. También las 'Metodologías del sueño'. Por ejemplo, cuando llegan apoyos a nuestras comunidades solamente el gobierno lleva sus proyectos. ¿Saben qué? Aquí tenemos pisos dignos o van a llegar viviendas. Y en la realidad de las comunidades tal vez no tengo piso para mí, pero es otra mi prioridad y eso ha sido muy significativo para mí porque uno u otra persona no puede saber lo que la otra necesita hasta que la misma persona lo diga: quiero o deseo esto. Entonces eso se trabaja a través de una metodología de acción participativa y a partir del sueño. Entonces es reunir a la comunidad, de generar confianza y que una vez generando confianza las mismas personas digan qué es lo que desean, qué es lo que sueñan y a partir de eso ver qué podemos hacer para que se llegue a lograr ese sueño (Teresa).

Teresa argumentó que planear los sueños o planeación con sujeto es trabajar con una metodología útil además de necesaria para subvertir el orden establecido frente al patriarcado que con sus actitudes canceló la posibilidad de planear los sueños de las mujeres:

Porque a veces vivimos la vida que no queremos. Vivimos una vida que otro quiso, que es mi papá, que es mi abuelo. Eso soñó él y eso hago, pero eso no me llena. Entonces es hacer lo que yo quiero, lo que yo deseo. Y si yo hago lo que quiero y lo que deseo lo voy a hacer bien y eso me va a hacer feliz (Teresa).

El testimonio de la entrevistada guarda relación con la filosofía que uno de los fundadores del CESDER, Benjamín Berlanga Gallardo, ha difundido profusamente: la planeación con sujeto a partir de sus sueños y propósitos. Las diversas experiencias con esta metodología han generado una corriente del pensamiento trascendente en la pedagogía del sujeto (Berlanga Gallardo, 2013).

Ahora bien, en esa reflexión para darnos cuenta del qué me-nos pasa se consideró pertinente cuestionar a los participantes del estudio acerca de qué les agrada del CESDER y estas fueron sus percepciones:

¿Qué me agrada? Desde la forma de organizarse para realizar las actividades. Desde que yo llegué me sorprendí; por ejemplo, la igualdad de los asesores, que uno no distingue quién es asesor y quién es alumno. No hay esa jerarquía al llegar, como mujer pues sí me impactó que el mismo director del CESDER está a mi lado lavando su vajilla, lavando su cuchara, secando, igual que todos nosotros. Ser hombre, ser director pues eso fuera de aquí (CESDER) está muy normalizado: yo soy el jefe y me hacen todo y aparte soy hombre. Las rotativas, todos vamos girando: todos pasamos por lavar vajillas, por hacer cena, por hacer desayuno. Todos aprendemos de todo. Las clases, las formas de los asesores. Por ejemplo, cuando nosotros iniciamos fue muy difícil y fuimos escuchados, a cada persona le dan como qué importancia. Nos decían también vamos a evaluar esfuerzos. Hay muchos factores que se conjugan acá y al venir de otro contexto miras muchos cambios (Teresa).

Las percepciones de Teresa van en el sentido de describir la atmósfera de trabajo en la institución educativa en un plano de horizon- 
Intentando Hacer Grietas en el Sistema Educativo Mexicano

talidad y equidad en la manera de administrar las relaciones de poder. En ese sentido, el mensaje es nos educamos en comunión haciendo trabajo colaborativo en una praxis socioeducativa que trasciende los linderos de los espacios áulicos (Messina Raymondi; Serrano Arroyo; Comunidad Aguilar, 2017; Berlanga Gallardo, 2020). En consecuencia, las labores de los estudiantes entrañan asumir compromisos éticos para cumplir responsabilidades que se nutren de la creación de conciencia en un proceso de aprendizaje en espiral que involucra ese darnos cuenta de lo que acontece (Berlanga Gallardo, 2013). Y es justamente ese darnos cuenta lo que conduce a los estudiantes del CESDER a problematizar los desajustes generados en el tiempo debido a la carencia de disciplina de las personas que no han asimilado la filosofía del trabajo colaborativo en las rotativas. Los argumentos esgrimidos por los estudiantes conducen a repensar las funciones que los miembros de una comunidad de aprendizaje deben asumir porque cada acción ilustra la diferencia que existe entre escolarizar y educarnos en comunión. Es crucial establecer esa distinción visibilizando la relación entre la reflexión y la acción en la compleja tarea de transformar actitudes y valores que ayuden a establecer sinergias. Así lo refiere Onésimo (22 años, promotor del desarrollo comunitario, Veracruz) sintetizando las percepciones que los siete sujetos informantes destacaron acerca de lo que acontece en el CESDER:

Lo que más me agradó es esa interacción con los compañeros porque es una comunidad de aprendizaje. Desde ahí ya está como rompiendo otros esquemas de la educación en donde todos aprendemos de todos. En realidad, aprendes charlando con los compañeros en el desayuno, en la comida, en los dormitorios.

Ahora bien, hay que decir lo que no me agrada es que hace algunos años el CESDER lo hablaban como una escuela diferente, una educación diferente. De unos años para acá como que ha empezado a tambalear y creo que es el proceso de la administración. Se necesita de una administración sólida en la que debe haber mucha comunicación porque ésta es muy importante para todos y ahí se tiene que checar un poquillo (Onésimo).

De acuerdo con lo comentado por Onésimo, la prueba del ácido de las instituciones y de los modelos educativos es la congruencia, transparencia y sostenibilidad de su estilo de gestión. Se trata de un estilo abierto y adaptativo a los cambios en el entorno para lo cual debe disponer de canales de comunicación receptivos de las resonancias que se generan en su hacer cotidiano (Berlanga Gallardo, 2013). Lo anterior se relaciona con la noción de Universidad efimera, con una vocación de servicio, orientada a generar e innovar su oferta de carreras, rediseñar sus planes y programas de estudios y actualizar su trato con las comunidades campesinas e indígenas en el territorio lo que coloca al CESDER en la ruta de la transformación permanente de sus procesos de gestión académica (Messina Raymondi; Serrano Arroyo; Comunidad Aguilar, 2017; Berlanga Gallardo, 2020). Desde esa visión de Universidad efímera se reconoce que los desafíos se agudizan por problemas educativos, de inseguridad alimentaria, pérdida de la biodiversidad, despojos territoriales, crisis de valores, impunidad, violencia cotidiana e institucional y la pandemia del coronavirus. La ocurrencia de estos fenóme- 
nos demanda más reflexión-acción sobre los modelos educativos y su permanente transformación para servir mejor a la sociedad (Berlanga Gallardo, 2020). Una muestra de lo anterior es el reciente rediseño de la Licenciatura en Planeación del Desarrollo Rural además de la redefinición de funciones, la creación de alianzas estratégicas y la cogeneración de propuestas de posgrados entre el CESDER y la Universidad Campesina Indígena en Red (UCI Red) para ofrecer los siguientes programas:

a) Licenciatura en Procesos Rurales Sustentables para una Vida Digna, con especialidades en:

Agroecología y seguridad alimentaria

Contabilidad y Administración en Organizaciones Sociales

Comunicación Social Comunitaria

Economía Social y Gestión de Emprendimientos Cooperativos

Procesos Alfareros e Innovación Tecnológica

b) Maestría en Pedagogía del Sujeto y Práctica Educativa

c) Maestría en Agroecología, Territorio y Soberanía Alimentaria

d) Maestría en Prácticas Narrativas en la Educación y el Trabajo Comunitario

e) Maestría en Educación con Personas Jóvenes y Adultas

f) Doctorado en Pedagogía del Sujeto

g) Doctorado Latinoamericano en Pedagogía Crítica [Proyecto]

h) Doctorado en Agroecología [Proyecto]

Es importante destacar que no obstante la crisis financiera que enfrenta la institución por la reducción presupuestaria de las agencias patrocinadoras en el ámbito internacional y la política de austeridad republicana ejecutada por el gobierno federal, el CESDER buscó financiamiento para cubrir el déficit de recursos mediante la realización de trabajos de consultoría para continuar ofreciendo educación asequible a la ciudadanía. La institución ha logrado costos de operación reducidos que cubren asesorías, material didáctico, alimentación y hospedaje en cada encuentro. A los asesores y profesores sólo se les apoya con los viáticos, la alimentación y el hospedaje en las instalaciones del CESDER en Zautla. Lo anterior es una muestra fehaciente del estilo de gestión guiado por las relaciones de confianza, cooperación y reciprocidad propias de una economía solidaria en la que se intercambia el trabajo docente como un acto de generosidad o donar un servicio a la comunidad de aprendizaje, evitando que la educación sea valorada como producción y eventualmente trocarse como una mercancía (González Forster, 2016; Berlanga Gallardo, 2020). Así, en todos los programas educativos se labora bajo la modalidad semi presencial y cada encuentro se convierte en una oportunidad de convivencia, conversatorio, actividades lúdicas y camaradería intercultural con personas que viajan periódicamente cada mes o trimestre, dependiendo del programa cursado- desde diversas regiones del país. En ese sentido, puede afirmarse que involucrarse en la misión y visión del CESDER implica una apuesta por la vida como 
Intentando Hacer Grietas en el Sistema Educativo Mexicano

afirma Hernández-Loeza (2014) en un texto que ilustra su decisión de involucrarse en un proyecto educativo congruente con su mirada sobre los modos de vida campesino e indígena en una región que necesita urgentemente de la sabiduría de los campesinos y de las aportaciones cognitivas de profesionales humanistas comprometidos en la formación de ciudadanos activos, informados y conscientes de la problemática de su territorio y del tiempo histórico que les ha correspondido enfrentar.

\title{
Discusión
}

Durante el mapeo de experiencias de aprendizaje en el CESDER se observó que existe una orientación clara hacia la formación crítica y propositiva de los sujetos sociales para crear consciencia de la compleja realidad que hoy enfrentan las comunidades rurales en el país. Al proceder así se está formando ciudadanía imbuyendo valores para el ejercicio de derechos y obligaciones orientados a establecer lazos de confianza y solidaridad informada para resistir a los embates de la modernidad sustentada en el extractivismo de los recursos naturales de las empresas transnacionales y la negación del modo de vida campesino (Berlanga Gallardo, 2016; CESDER, 2017). En la praxis es la búsqueda de lucro de las empresas tuteladas por el Estado a costa de la vida en los territorios afectados por la ejecución de estos proyectos alrededor del mundo. Al respecto, Tetreault (2015, p. 254) destaca que:

\begin{abstract}
El crecimiento del sector ha sido espectacular a lo largo de los últimos 15 años en el contexto de las reformas de libre mercado orientadas a la atracción de Inversión Extranjera Directa (IED), y alentado por el alza en el precio de los minerales. Durante este tiempo, el gobierno mexicano ha repartido cientos de concesiones mineras a compañías extranjeras, principalmente canadienses. Esas compañías -que cuentan con tecnología de punta, son altamente eficientes en términos económicos y resultan totalmente destructivas en términos ecológicos- han podido extraer desde el año 2000 el doble de oro y la mitad de plata del país que lo que se extrajo en todo el periodo de trescientos años de conquista y colonialismo [Fernández Vega, 2011]. También se están extrayendo otros minerales a un ritmo acelerado.
\end{abstract}

El autor hace referencia a las luchas de resistencia desplegadas por los diversos grupos humanos cuyas vidas fueron afectadas por las concesiones del gobierno federal a grupos de inversionistas y estas acciones vulneraron los intereses de los grupos marginados al crear las condiciones administrativas y legales para el arribo de las empresas mineras.

Ahora bien, para examinar un entorno dominado por los proyectos extractivistas que atentan contra la vida se requiere de un modelo educativo que promueva el debate y la reflexión comprometida y ese desafío lo asume el CESDER desde la educación popular para cuestionar sobre la vida. Las reflexiones de Berlanga Gallardo (2013) cultivan la mirada crítica y emancipadora: 
Imaginemos que la educación no es primero conocer qué es la Vida, sino, ante todo saber cómo nos va en la vida: que en la educación tomamos el camino largo de la experiencia para hacer aprendizajes, en lugar del camino de la explicación, de la demostración. Se trata de apurar un atrevimiento: de tocar aquello en torno a lo que gira todo el ordenamiento educativo, el logos.

Suspender el conocimiento de lo que es, de la Verdad y el Deber Ser, para encontrarnos en el padecer, en la incertidumbre del ir siendo: buscar que lo educativo se ordene en torno a la contingencia, al asombro y pasmo del ir siendo y no en torno a la "fría y desangelada" imagen del ser, de lo que es, de la verdad escrita, legislada, establecida. Al hacerlo, al intentar este giro epistémico, esta otra manera de ponernos ante el mundo para saber de él, salimos de la seguridad y del refugio de la razón para aventurarnos a nombrar la incertidumbre del acaecer, del ir siendo: nos asomamos a la densidad de la propia vida y a la densidad de lo que vamos viviendo en común, como motivo distintivo del educar (Berlanga Gallardo, 2013, p. 46-47).

Esa forma de enfocar cómo nos va en la vida parte de una mirada crítica y profunda que implica un darnos cuenta de nuestra condición humana sometida a las asimetrías en el ejercicio del poder en el hogar, la escuela, el centro de trabajo y en las relaciones de la sociedad civil con el Estado.

Por otra parte, quienes incursionaron en el estudio de la experiencia de las redes educativas del CESDER han documentado el posicionamiento ético político de sus docentes y las actitudes de sus comunidades de aprendizaje (Izquierdo Moreno, 2010; González Forster, 2016; Messina Raymondi; Serrano Arroyo; Comunidad Aguilar, 2017; Hernández-Loeza, 2014; 2018). Así, dependiendo del enfoque que una Universidad adopte y de la congruencia de su praxis, la educación puede constituirse en un medio para la transformación de la realidad o devenir en un instrumento para reproducir las condiciones de dominio y opresión contra los pueblos (Berlanga Gallardo, 2011; 2013; Velasco Cruz, 2016). En consecuencia, cuando se elige transitar para subvertir el orden en el sistema educativo, en el CESDER se busca superar las modas administrativas adoptadas por las Universidades latinoamericanas cuestionadas por Quintar (2019, p. 253):

No olvidemos que las universidades eran parte del desarrollo nacional, sobre todo durante el siglo XX, y fueron, desde el siglo XIX, siglo de las luces, el lugar privilegiado de la generación de pensamiento y referencia en la reflexión social.

Actualmente pareciera que este lugar se ha ido disolviendo en la maraña de las tensiones financieras y de desactualización en relación con la época y sus exigencias, emergiendo un proceso de banalización del trabajo académico debido a un sobredimensionamiento de las labores administrativo-financieras, lo que se expresa en pérdida de calidad, a pesar de los indicadores 'de calidad', 
Intentando Hacer Grietas en el Sistema Educativo Mexicano

al modo de las famosas normas ISO, que en la mayoría de las ocasiones deshistorizan y siempre parametralizan el hacer universitario.

La autora hace énfasis en que el proceder estandarizado de las entidades educativas les permite agenciarse fondos provocando el beneplácito de las autoridades universitarias porque se cumplieron las metas impuestas desde el Estado y organismos financieros multinacionales pero ese procedimiento no garantiza que las Instituciones de Educación Superior (IES) estén respondiendo a las necesidades culturales de la sociedad. En consecuencia, se busca vender imagen y una cultura de la simulación contraria a la misión de imbuir valores en el sujeto social para transformar su realidad (Quintar, 2019; Ortiz Palomeque; Arcos López, 2020). Es precisamente esta situación en la que se encuentran las IES en el país lo que inspiró en los promotores del CESDER procesos de reflexión que derivaron en la decisión por un enfoque alternativo para la formación de ciudadanos activos que al integrarse en comunidades de aprendizaje conversen, intercambien información, cobren conciencia, se organicen y encaren los desafíos de la modernidad excluyente (Berlanga Gallardo, 2011; 2013; Hernández-Loeza, 2014). Esta modernidad se materializa a través de proyectos de muerte que trastocan ancestrales modos de vida, provocan que los campesinos vean su existencia en el territorio como vidas precarias que no vale la pena vivir (Berlanga Gallardo, 2018). En síntesis, estos proyectos de muerte tutelados desde el Estado atentan contra la biodiversidad de los territorios y saquean la riqueza de su memoria biocultural (Berlanga Gallardo, 2011; Toledo; Barrera Bassols, 2008; CESDER, 2017).

\section{Conclusiones}

En este recorrido por el CESDER, atendiendo a las voces de algunos de los estudiantes en el nivel de licenciatura, se constató que la institución educativa ha influido de manera favorable en sus modos de vida. Y esto es así porque en el modelo implementado se invierte el proceso de aprendizaje desde la praxis in situ a la teoría (Evelia, Dámaso, Teresa y Onésimo). Las experiencias en sus comunidades son reflexionadas y resignificadas en cada encuentro mensual para generar comunidades de aprendizaje que implica donar experiencias en cada encuentro solidario con los otros (Grecia y Sammy). En el CESDER se vive con agrado cada encuentro porque es enriquecedor.

Sin embargo, destacaron que estos encuentros han transitado por un proceso de aprendizaje complejo que obliga a autorregularse (Teresa y Onésimo). Estas experiencias pedagógicas potencian nuestras prácticas, conocimientos y metodologías para incidir en la defensa de los territorios campesinos y la soberanía alimentaria (Teresa y Dámaso).

El modelo pedagógico que considera el círculo virtuoso de la acción-reflexión-acción resignificada de los sujetos sociales preserva la cultura política de la resistencia, los modos de vida buena para consolidar los lazos de confianza y solidaridad en el contexto de la agricultura de la 
pobreza. Para cumplir con esa misión, el CESDER se gestiona como un organismo social adaptativo o Universidad efimera, generando alianzas con diversos actores e instituciones, innovando programas híbridos de formación técnica, ética y política que coadyuven a una cultura cívica para subvertir el orden en un camino sinuoso.

Recibido el 1 de junio de 2021 Aprobado el 9 de agosto de 2021

\section{Referencias}

AYUSTE, Ana; GROS, Begoña; VALDIVIELSO, Sofía. Sociedad del conocimiento: Perspectiva pedagógica. En: GARCÍA ARETIO, Lorenzo (Ed.). Sociedad del conocimiento y educación. Madrid: UNED, 2012. P. 17-40.

BERLANGA GALLARDO, Benjamín. Universidad y sociedad civil: encontrar los modos de una relación posible y necesaria ante los que nos pasa, ante lo que acontece. Zautla: UCI Red CESDER, 2011. Disponible en: http://www.ucired. org. $\mathrm{mx} /$ index.php/publicaciones/pedagogía-sujeto/fundamntos/item/3-universidad sociedad-civil. Consultado en: 15 dic. 2020.

BERLANGA GALLARDO, Benjamín. ¿Volver al sujeto? Once ideas para pensar la educación popular como una apuesta radical, de resistencia, para hacernos sujetos de la digna rabia. La Piragua, Revista Latinoamericana y Caribeña de Educación y Política, Lima, n. 38, p. 41-50, oct., 2013. Disponible en: http://www. ipc.org.co/agenciadeprensa/wp-content/uploads/2014/02/Docto115-RevistaLa-Piragua-CEEAL-Movimientos-Sociales-y-Educaci\%C3\%B3n-Popular.pdf. Consultado en: 11 abr. 2021.

BERLANGA GALLARDO, Benjamín. Del campesino desposeído a la desposesión campesina la construcción social de las vidas campesinas como vidas que no merecen la pena ser lloradas y las luchas campesinas por habitar de modo propio el mundo. Zautla: UCI Red CESDER, 2018. Disponible en: https://www. academia.edu/37863917/DEL_CAMPESINO_DESPOSEIDO_A_LA_DESPOSESION_CAMPESINA. Consultado en: 10 mayo 2021.

BERLANGA GALLARDO, Benjamín. Cinco ideas sobre la profundización del apagón pedagógico en tiempos de pandemias: Los retos de la pedagogía del sujeto. Zautla: UCI Red-CESDER, 2020. Disponible en: https://www.academia. edu/43766245/APAGON_PEDAGOGICO. Consultado en: 18 mayo 2021.

CASTELLS, Manuel; HIMANEN, Pekka (Coord.). Modelos de desarrollo en la era global de la información. México: FCE, 2016.

CESDER. Minería Canadiense en Puebla y su impacto en los Derechos Humanos: Por la vida y el futuro de Ixtacamaxtitlán y la Cuenca del Río Apulco. Puebla: PODER; CESDER PRODES; IMDEC; Unión de Ejidos y Comunidades en Defensa de la Tierra, el Agua y la Vida, Atcolhua, 2017.

CHOMSKY, Noam; PAPPÉ, Ilan; BARAT, Frank. On Palestine. New York: Penguin Books, 2015.

CHOMSKY, Noam; PAPPÉ, Ilan. Conversaciones sobre Palestina. Buenos Aires: Marea Editorial, 2016.

DIETZ, Gunther. Multiculturalismo, interculturalidad y diversidad en educación: una aproximación antropológica. México: FCE, 2012.

DIETZ, Gunther; ÁLVAREZ VEINGER, Aurora. Reflexividad, interpretación y colaboración en etnografía: un ejemplo desde la antropología de la educación. En: 
Intentando Hacer Grietas en el Sistema Educativo Mexicano

OEHMICHEN BAZÁN, Cristina (Ed.). La etnografía y el trabajo de campo en las Ciencias Sociales. México: UNAM Instituto de Investigaciones Antropológicas, 2014. P. 55-89.

DURAND PONTE, Víctor Manuel. La cultura política de los mexicanos en el régimen neoliberal. En: RODRÍGUEZ ARAUJO, Octavio (Coord.). México ¿Un nuevo régimen político? México: Siglo XXI Editores, 2009. P. 121-149.

DUSSEL, Enrique. 14 tesis de ética: Hacia la esencia del pensamiento crítico. México: Editorial Trotta, 2016.

EVANS, Brad; REID, Julian. Una vida en resiliencia: El arte de vivir en peligro. México: FCE, 2016.

GARCÍA CARRASCO, Joaquín et al. Nuevos modos de aprendizaje en el contexto de la Sociedad del conocimiento. En: GARCÍA ARETIO, Lorenzo (Ed.). Sociedad del conocimiento y educación. Madrid: UNED, 2012. P. 305-339.

GONZÁLEZ FORSTER, Sara Lúa. No hacemos al otro, nos hacemos juntos: miradas desde la antropología a la red educativa del CESDER en la sierra norte de Puebla. Cuadernos del Sur, Oaxaca, v. 21, n. 41, p. 62-77, 2016.

HERNÁNDEZ-LOEZA, Sergio Enrique. El CESDER y la UCIRED como apuestas de vida en la formación de sujetos de la digna rabia desde Instituciones de Educación Superior (IES) en México. Revista Uturunku Achachi, Chile, v. 3, p. 45-67, 2014. Disponible en: https://www.uturunkuachachi.academialatinoamericana.org. Consultado en: 18 dic. 2020.

HERNÁNDEZ-LOEZA, Sergio Enrique. Educación superior, diversidad cultural e interculturalidad en América Latina. Estudio sobre México. En: MATO, Daniel (Coord.). Educación superior, diversidad cultural e interculturalidad en América Latina. Córdoba: UNESCO-IESALC-UNC, 2018. P. 225-248.

HERNÁNDEZ-LOEZA, Sergio; MANJARREZ MUÑOZ, Yunuen. La Universidad Campesina Indígena en Red (UCIRED). En: MATO, Daniel (Coord.). Educación superior y pueblos indígenas en América Latina: experiencias, interpelaciones y desafíos. México: Universidad Nacional de Tres de Febrero; Universidad Nacional Autónoma de México, 2016. P. 261-280.

IZQUIERDO MORENO, Eduardo. Alternativas al desarrollo en zonas rurales de extrema pobreza: La experiencia del Centro de Estudios para el Desarrollo Rural (CESDER) en la Sierra Norte de Puebla. 2010. 250 p. Tesis (Maestría en Ciencias en Planificación) - Programa de Maestría en Ciencias en Planificación, Instituto Politécnico Nacional, México, 2010.

MESSINA RAYMONDI, Graciela; SERRANO ARROYO, Ignacia; COMUNIDAD AGUILAR, Marco Antonio (Coord.). ¿Qué formación? Relato desde historias de vida: La experiencia del CESDER. Puebla: Fundación Intered; Agencia Vasca de Cooperación para el Desarrollo, 2017.

ORTIZ PALOMEQUE, Germán; ARCOS LÓPEZ, Nicolás. Vinculando prácticas discursivas, vivencias y saberes en la Universidad Intercultural del Estado de Tabasco. Cuadernos del Instituto Nacional de Antropología y Pensamiento Latinoamericano, Buenos Aires, v. 29, n. 1. 2020. Disponible en: http://revistas. inapl.gob.ar/index.php/cuadernos/article/view/1104. Consultado en: 10 mayo 2021.

PAPALIA, Diane E.; WENDKOS OLDS, Sally; DUSKIN FELDMAN, Ruth. Psicología del desarrollo: De la infancia a la adolescencia. 11. ed. México: McGraw Hill, 2019. 
PAPPÉ, Ilan. La idea de Israel: Una historia de poder y conocimiento. Madrid: Akal, 2015.

PAPPÉ, Ilan. La cárcel más grande de la tierra: Historia de los territorios ocupados. Madrid: Capitán Swing, 2018.

QUINTAR, Estela. La universidad latinoamericana: Entre el sujeto interpretado y las coordenadas de lo posible. En: BASAIL RODRÍGUEZ, Alain (Coord.). Academias asediadas: Convicciones y conveniencias ante la precarización. Buenos Aires: CLACSO; Tuxtla Gutiérrez: CESMECA-UNICACH, 2019. P. 247-282.

ROCKWELL, Elsie. La experiencia etnográfica: Historia y cultura en los procesos educativos. Buenos Aires: Paidós, 2009.

SCHUNK, Dale. Teorías del aprendizaje: Una perspectiva educativa. 6. ed. México: Pearson Educación, 2012.

SUMOANO, María Fernanda; NIETO, Fernando. Ciudadanía en México: ¿Ciudadanía activa? México: COLMEX-INE, 2015.

TETREAULT, Darcy V. México: la ecología política de la minería. En: VELTMEYER, Henry; PETRAS, James (Coord.). El neoextractivismo: ¿Un modelo posneoliberal de desarrollo o el imperialismo del siglo XX? México: Edit. Crítica, 2015. P. 253-279.

TOLEDO, Víctor Manuel; BARRERA BASSOLS, Narciso. La memoria biocultural: La importancia ecológica de las sabidurías tradicionales. Barcelona: Icaria Editorial, 2008

TURBINO, Fidel. La interculturalidad en cuestión. Lima: Pontificia Universidad Católica de Perú Fondo Editorial, 2016

VELASCO CRUZ, Saúl. Proyecto educativo y movilización política: El caso del CESDER en la sierra norte de Puebla. En: OLIVIER TÉLLEZ, María Guadalupe (Coord.). Educación, política y movimientos sociales. México: UAM; CONACYT; Edit. Colofón; Red Mexicana de Estudios de los Movimientos Sociales, 2016. P. 177-202.

VELTMEYER, Henry; PETRAS, James (Coord.). El neoextractivismo: ¿Un modelo posneoliberal de desarrollo o el imperialismo del siglo XX? México: Edit. Crítica, 2015.

ZEMELMAN, Hugo. Voluntad de conocer: El sujeto y su pensamiento en el paradigma crítico. México: Editorial Anthropos; UNACH, 2005.

Germán Ortiz Palomeque, Maestría en Ciencias en Producción Agroalimentaria en el Trópico, Docente de Tiempo Completo, Universidad Intercultural del Estado de Tabasco, Oxolotán, Municipalidad de Tacotalpa, Tabasco, México.

ORCID: https://orcid.org/0000-0001-9995-1284

E-mail: georpa36@hotmail.com

Editor a cargo: Luís Armando Gandin

Este é um artigo de acesso aberto distribuído sob os termos de uma Licença Creative Commons Atribuição 4.0 Internacional. Disponível em: <http:// creativecommons.org/licenses/by/4.0>. 